\title{
Bone marrow metastasis of prostatic adenocarcinoma with post-treatment foamy-like changes: A diagnostic pitfall
}

\author{
Nuri Yigit, MD; ${ }^{*}$ Julia Turbiner Geyer, $M D^{+}$
}

*Department of Pathology and Laboratory Medicine, Weill Cornell Medical College/New York-Presbyterian Hospital, New York, NY; and Department of Pathology, Gulhane Military Medical Academy and School of Medicine, Ankara, Turkey; †Department of Pathology and Laboratory Medicine, Weill Cornell Medical College/New York-Presbyterian Hospital, New York, NY

Cite as: Can Urol Assoc J 2014;8(11-12):e941-3. http://dx.doi.org/10.5489/cuaj.2402

Published online December 15, 2014.

\section{Abstract}

Prostatic acinar adenocarcinoma (PAA) is the most common malignant tumour of the male genital system. Hormonal therapy and radiotherapy are widely-used treatment modalities in advanced stage disease. However, with hormonal therapy, the morphology of the neoplastic cells can be altered with a shift towards benign-appearing cells with inconspicuous nucleoli and abundant cytoplasm. When the tumour spreads to the bone marrow, these neoplastic cells can mimic foamy histiocytes, which may lead to a misdiagnosis. This becomes more challenging if a concurrent bone marrow malignancy is identified. Attention should be paid if foamy cells are detected in bone marrow, particularly if the patient has a history of PAA. To the best of our knowledge, this is the first report of simultaneous bone marrow involvement by metastatic PAA with mimicking foamy histiocytes and lymphoplasmacytic lymphoma.

\section{Introduction}

Prostatic acinar adenocarcinoma (PAA) is the most common malignant tumour of the male genital system. ${ }^{1}$ Androgen deprivation therapy (ADT), a mainstay for treatment of advanced PAA, can alter tumour cell morphology, with changes reminiscent of foamy macrophages. ${ }^{2}$ These bland morphologic features create a challenge in rendering a correct diagnosis of PAA, particularly when a distant metastatic lesion is biopsied. Foamy cells in the bone marrow are usually construed as histiocytes - innocent bystanders and not primary causal agents in bone marrow pathology. Typically, the presence of bland-appearing foamy cells does not require further investigation with auxiliary studies. As a consequence, metastatic malignant cells with similar morphology can be overlooked.

\section{Case report}

An 83-year-old man presented with new anemia with a hemoglobin level of $8.9 \mathrm{~g} / \mathrm{dL}$, increased serum IgM level of $2340 \mathrm{mg} / \mathrm{L}$ (normal range: 56-352 mg/dL), free kappa light chain level of $7.99 \mathrm{mg} / \mathrm{dL}$ (normal range: $0.33-1.94 \mathrm{mg} / \mathrm{dL}$ ), and $M$ protein at a concentration of $1.3 \mathrm{~g} / \mathrm{dL}$. Consequently, bone marrow biopsy was performed to further evaluate the monoclonal gammopathy. The patient had a medical history of melanoma 14 years ago, as well as PAA diagnosed 2 years ago. At the time of the PAA diagnosis, his prostatespecific antigen (PSA) level was $86.2 \mathrm{ng} / \mathrm{mL}$ (normal range: $<4 \mathrm{ng} / \mathrm{mL}$ ), bilateral core biopsies showed conventional PAA and Gleason score of $9(4+5)$ involving each one of the 12 cores (Fig. 1, part A). The tumour had no evidence of foamy gland carcinoma, even partially. The patient was considered inoperable and hormonal therapy was administered with luteinizing hormone-releasing hormone blockers - monthly degarelix followed by leuprolide injections. His most recent laboratory studies showed a PSA value of $0.44 \mathrm{ng} / \mathrm{mL}$.

\section{Histopathologic examination}

At the low power, the deeper half of the biopsy consisted of an admixture of small mature-appearing lymphocytes, plasmacytoid lymphocytes, and many mature plasma cells some of which included large intranuclear pseudoinclusions (Dutcher bodies) (Fig. 1, parts B, C). On the other hand, the subcortical half of the biopsy was occupied by large aggregates of monotonous bland-appearing large cells which contained mainly clear and rarely eosinophilic foamy cytoplasm, distinct cytoplasmic membrane, medium-sized, and round eccentric nuclei with chromatin clumping and inconspicuous nucleoli (Fig. 1, part D). Subtle focal intrasinusoidal collections of the cells, active bone remodelling and bone marrow fibrosis were also noted in these subcortical areas. 


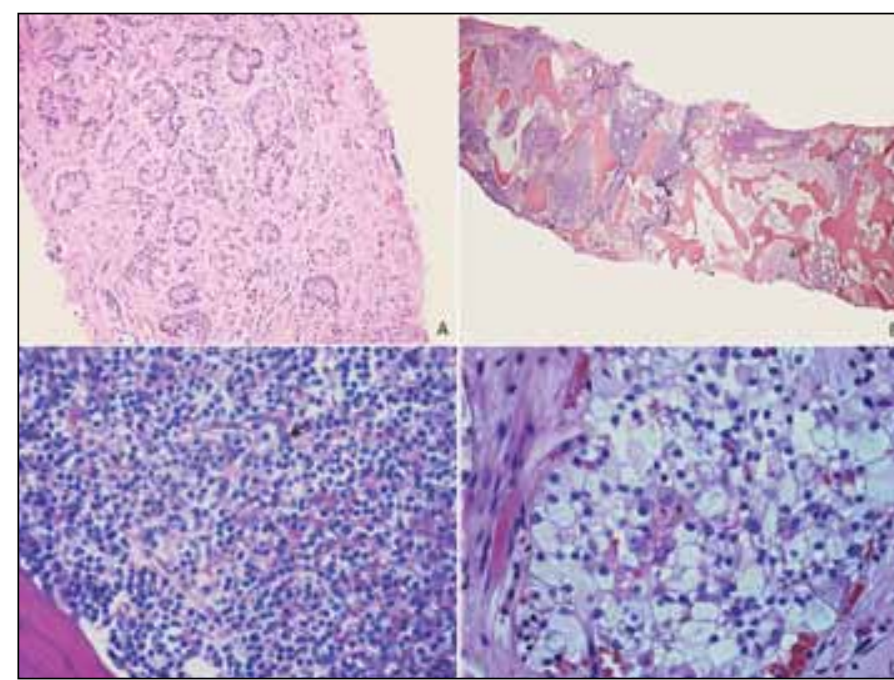

Fig. 1. A. Prior prostate core specimens include neoplastic glands that are composed of mostly columnar cells with compact eosinophilic cytoplasms, large nuclei and prominent nucleoli (hematoxylin and eosin stain [H\&E] $\times 40$ ). $B$. Bone marrow involvement by lymphoma on the left side and metastatic foci of prostate carcinoma on the right (arrows). Active bone remodeling is seen around the foamy-appearing tumour cells $(H \& E, \times 200)$. C. Lymphoplasmacytic lymphoma/Waldenström macroglobulinemia infiltration is seen as a mixture of small lymphocytes, many of them plasmacytoid, and plasma cells with Dutcher bodies (arrow) $(H \& E, \times 400)$. D. A large tumour embolus, composed of the prostatic carcinoma cells with foamy-like changes, is seen in the bone marrow sinus. Tumour cells' cytoplasms are mostly clear and rarely contain eosinophilic vacuoles $(H \& E, \times 400)$.

The immunohistochemical studies revealed extensive bone marrow infiltration by CD20-positive B-lymphocytes and numerous admixed CD138-positive plasma cells with kappa light chain restriction (Fig. 2, part A1-3). On the other hand, the foamy-appearing cells were diffusely positive for AMACR and CAM5.2 (dim staining), strongly positive for prostate-specific membrane antigen (PSMA), and very focally weakly positive for PSA, PSA phosphatase (PSAP), and pancytokeratin (Fig. 2, B1-3). CD68, ERG, P501S, CK7, PAX8, CD10 and S100 were all negative.

Thus, a diagnosis of synchronous metastatic PAA with post-treatment changes and lymphoplasmacytic lymphoma/ Waldenström macroglobulinemia (LPL/WM) was rendered.

\section{Discussion}

Bone is by far the most frequent site of PAA metastasis $(90 \%){ }^{3}$ Radiotherapy and hormonal therapy are the most common modalities in advanced stage PAA. Both therapy strategies can change tumour cell morphology, which is mostly foamy-like., ${ }^{4,5}$

The main differential diagnosis is with foamy macrophages, which are frequently seen in various marrow disorders including hematologic neoplasms, storage diseases, and infections. Especially, their presence in bone marrow

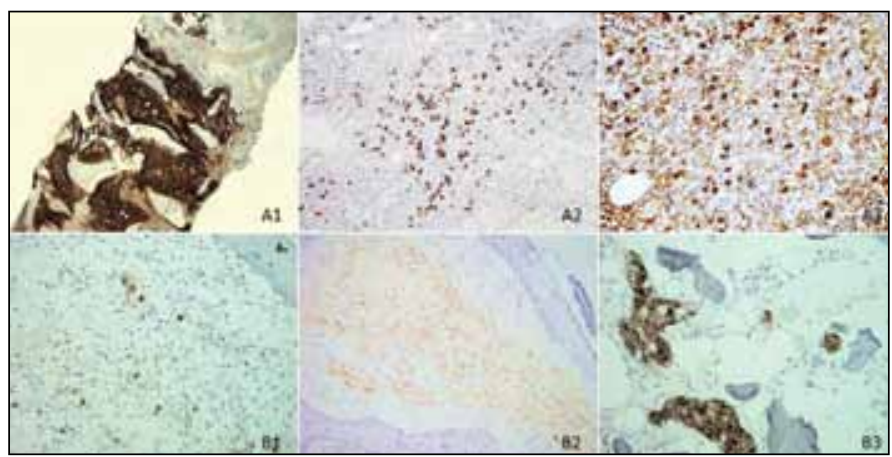

Fig. 2. A1, CD20 reveals extensive infiltration of $B$ cell lymphoma (immunoperoxidase, $\times 40$ ). A2 and A3. Monotypic plasma cell component of lymphoma are proved by CD138 and Kappa light chain antibody (immunoperoxidase, $A 2, \times 100 ; A 3, \times 200$ ). B1. Neoplastic foamy-appearing cells shows only focal expression of prostate-specific antigen (immunoperoxidase, $\times 200$ ). B2. Mild and diffuse AMACR positivity is seen in nearly all neoplastic cells (immunoperoxidase, $\times 200$ ). B3. Strong prostate-specific membrane antigen marking reveals their prostatic origin (immunoperoxidase, $\times 200$ ).

biopsies typically reflects the overall acceleration of the cell turnover, seen in various conditions, such as chronic myelogenous leukemia, myelodysplastic syndrome, lymphoma, and immune thrombocytopenic purpura. ${ }^{6,7}$ Also, post-chemotherapy bone marrow biopsies are expected to contain phagocytic foamy histiocytes, which are in charge of removing necrotic debris prior to the anticipated regeneration of the bone marrow. Additionally, certain non-hematologic neoplasms including balloon-cell melanoma can exhibit foamy morphology. ${ }^{8}$

Considering all types of foamy cells, a thorough examination of the routine hematoxylin and eosin (H\&E) stained section can provide the following key findings:

- Foamy histiocytes generally have small, oval or indented, usually centrally located nuclei with finely dispersed chromatin and indistinct nucleoli, while malignant foamy cells are larger than histiocytes and contain round, hyperchromatic nuclei with occasionally prominent nucleoli.

- Foamy histiocytes in infectious diseases frequently contain numerous microorganisms that can be visible with routine H\&E stain (e.g., leishmaniasis and histoplasmosis).

- Foamy histiocytes are generally seen as scattered individual cells intermingled within the maturing hematopoietic elements and may sometimes form small loose clusters with ill-defined margins, but rarely make well-defined compact granulomas. Carcinoma cells tend to be found in larger discrete aggregates that are generally separate from hematopoietic elements.

- Histiocytes are not present in circulation. Therefore, the presence of intrasinusoidal collections of foamy cells implies tumor emboli. 
- Foamy histiocytes do not disturb bone marrow architecture. Neoplastic cells are almost always associated with fibrotic stroma and frequently cause bone remodeling and new bone formation.

This report emphasizes that the expression of the commonly used PAA markers, like pancytokeratin, PSA and PSAP, may be lost or sustained only focally in treated, advanced or poorly differentiated PAA. ${ }^{9}$ Conversely, up-regulation of PSMA was demonstrated in the more aggressive and hormone independent prostate cancer cells. ${ }^{10}$ Expression of AMACR may be seen in other types of carcinoma. Thus, an extensive immunohistochemical panel, especially including PSMA, is frequently needed to make a diagnosis of metastatic PAA. These markers help in the differential diagnosis with foamy histiocytes, as they are routinely negative. CD68 and CD163 are consistently positive in macrophages and are negative in carcinoma cells. Lack of S100 protein expression excludes the vast majority of melanomas including ballooncell variant.

The incidence of LPL/WM and prostate cancer in United States is 0.35 and 153 per 100000 people and men, respectively. ${ }^{11}$ There is no definite evidence of concurrent occurrence of LPL/WM and prostate carcinoma metastasis in bone marrow. Therefore, it is likely that in this case, the association of these two neoplasms in the bone marrow is coincidental. To our best knowledge, a case of bone marrow metastasis of PAA with post-treatment foamy-like changes and LPL/WM has not been reported to date in the Englishlanguage literature.

\section{Conclusion}

In male patients, the presence of aggregates of blandappearing foamy cells in bone marrow biopsy should raise a possibility of PAA metastasis with post-treatment changes. The expression of the commonly used PAA immunohistochemical markers PSA, PsAP, P501S, ERG and pancytokeratin may be decreased or lost in poorly-differentiated tumours, advanced stage disease, and/or effects of bone marrow decalcification procedure. Moreover, the positivity for AMACR is not observed in all PAA cases and can be seen in other types of carcinomas. Thus, an extensive immuno- histochemical panel may be required to make a diagnosis of metastatic PAA. Communication with the treating physician and any disclosure of PAA or ADT history are essential.

Acknowledgement: We thank Juan Miguel Mosquera, MD, MSc, for his valuable review of the manuscript.

Competing interests: Authors declare no competing financial or personal interests.

This paper has been peer-reviewed.

\section{References}

1. Siegel R, Ma J, Zou Z, et al. Cancer statistics, 2014. CA Cancer J Clin 2014;64:9-29. http://dx.doi. org/10.3322/caac.21208

2. Kitagawa Y, Koshida K, Mizokami A, et al. Pathological effects of neoadjuvant hormonal therapy help predict progression of prostate cancer after radical prostatectomy. Int J Urol 2003;10:377-82. hittp:// dx.doi.org/10.1046/i.1442-2042.2003.00640.x

3. Krishnan C, George TI, Arber DA. Bone marrow metastases: A survey of nonhematologic metastases with immunohistochemical study of metastatic carcinomas. Appl Immunohistochem Mol Morphol 2007;15:1-7. http://dx.doi.org/10.1097/01.pai.0000213134.09160.14

4. Gaudin PB. Histopathologic effects of radiation and hormonal therapies on benign and malignant prostate tissues. J Urol Pathol 1988:855-67.

5. Civantos F, Marcial MA, Banks ER, et al. Pathology of androgen deprivation therapy in prostate carcinoma. A comparative study of 173 patients. Cancer 1995;75:1634-41. http://dx.doi.org/10.1002/10970142(19950401)75:7<1634::AID-CNCR2820750713>3.0.C0;2-\#

6. Thiele J, Schmitz B, Fuchs R, et al. Detection of the bcr/abl gene in bone marrow macrophages in CML and alterations during interferon therapy-a fluorescence in situ hybridization study on trephine biopsies. J Pathol 1998;186:331-5. http://dx.doi.org/10.1002/(SICI) 1096-9896(199811)186:3<331::AIDPATH178>3.0.C0;2.7

7. Vaughan WP, Karp JE, Burke PJ. Effective chemotherapy of acute myelocytic leukemia occurring after alkylating agent or radiation therapy for prior malignancy. J Clin Oncol 1983;1:204-7.

8. Naresh KN, Lampert I, Hasserijan R, et al. Optimal processing of bone marrow trephine biopsy: The Hammersmith Protocol. J Clin Pathol 2006;59:903-11. http://dx.doi.org/10.1136/icp.2004.020610

9. Wright $\mathrm{GL} \mathrm{Jr}$, Grob BM, Haley C, et al. Upregulation of prostate-specific membrane antigen after androgendeprivation therapy. Urology 1996;48:326-34. http://dx.doi.org/10.1016/S0090-4295(96)00184-7

10. National Cancer Institute. Cancer Query Systems: Delay-Adjusted SEER Incidence Rates. http://surveillance. cancer.gov/delay/canques.html. Accessed December 11, 2014.

11. Cassetti T, Stracci F, Minelli L, et al. Metachronous malignancies in men with previous prostate cancer in Umbria, Italy, 1994-2003. Tumori 2008;94:7-10.

Correspondence: Dr. Nuri Yigit, Department of Pathology and Laboratory Medicine, Weill Cornell Medical College/New York-Presbyterian Hospital, 525 East 68th Street Starr Pavilion, 715 New York, NY 10065; nuy2001@med.cornell.edu; nyigit@gata.edu.tr 